\title{
Effect of donor kidney morphology parameters on the prognosis in living kidney transplantation recipients
}

\author{
Yang Qiu, Jinpeng Liu, Yamei Jiang, Turun Song, Zhongli Huang, Yu Fan, Xianding Wang, Tao Lin \\ Department of Urology/Institute of Urology/Organ Transplantation Center, West China Hospital, Sichuan University, Chengdu, China \\ Contributions: (I) Conception and design: Y Qiu, J Liu; (II) Administrative support: X Wang, T Lin; (III) Provision of study materials or patients: Z \\ Huang, X Wang, T Lin; (IV) Collection and assembly of data: Y Qiu, J Liu, Y Jiang; (V) Data analysis and interpretation: Y Qiu, T Song, Z Huang, \\ Y Fan; (VI) Manuscript writing: All authors; (VII) Final approval of manuscript: All authors. \\ Correspondence to: Xianding Wang, MD; Tao Lin, MD. Department of Urology/Institute of Urology/Organ Transplantation Center, West China \\ Hospital, Sichuan University. Number 37, Guoxue Alley, Chengdu 610041, China. Email: kidney1234@163.com.
}

\begin{abstract}
Background: The effect of donor kidney morphology parameters on the prognosis of renal transplant recipients remains unclear.

Methods: We conducted a retrospective cohort study consisting of 290 pairs of donors and recipients who underwent living related renal transplantation in our center between December 2013 and December 2015. The donor kidney morphology parameters, demographic characteristics and renal function of the included participants were collected and analyzed.

Results: The univariate linear regression analysis revealed that the donor kidney weight (DKW)/recipient body weight (RBW), DKW/recipient body surface area (RBSA), DKW/recipient body mass index (RBMI), donor kidney volume (DKV)/RBW, DKV/RBSA, DKV/RBMI, and donor body weight (DBW)/RBW were significantly correlated with estimated glomerular filtration rate (eGFR) and serum creatinine in recipients within two years of transplantation. In our multivariate linear regression analysis, DKW/RBW and donor age significantly correlated with eGFR at 6,12, 18 and 24 months after transplantation. DKW/RBW had the best prediction performance for good renal allograft function at 12 months after transplantation. We found that when the age of the donor was 55 years and above, the prediction performance of the nephron dosing to the recipient's metabolic demands mismatch was elevated. After grouping according to the donor's age and DKW/RBW, the allograft function from different donor ages improved as DKW/RBW increased. The number of recipients who were rejected in the high DKW/RBW group was significantly fewer than those in the low and medium groups the 1,2, and 3-year graft and patient survival rates were comparable among these groups. Furthermore, we also identified that when the age of the donor was 55 years and above, the threshold of DKW/RBW was $3.09 \mathrm{~g} / \mathrm{kg}$ via the receiver operating characteristic curve, which predicted a good renal function 12 months after transplantation.
\end{abstract}

Conclusions: The donor kidney morphology parameters were significantly associated with early renal allograft function, especially when the age of the donor was 55 years and above.

Keywords: Donor kidney; living; morphology parameters; renal transplantation

Submitted Feb 28, 2020. Accepted for publication Aug 19, 2020.

doi: 10.21037/tau-20-680

View this article at: http://dx.doi.org/10.21037/tau-20-680

\section{Introduction}

Kidney transplantation is the best treatment for end-stage renal disease (ESRD) because it has a better life quality and less cost when compared with dialysis patients $(1,2)$. Due to the shortage of donor kidneys, living kidney transplantation has been widely used throughout in the world (3-5). After decades of effort, the short-term survival of kidney allografts and recipients has significantly increased, nevertheless, 
the long-term outcomes remain subject to further improvement (6).

Due to the improvement of immunosuppressants and induction therapies, more focus is placed on the nonimmunological factors. Previous animal studies have indicated that kidney mass loss can result in a chronic injury of the remaining kidney (7-10). Brenner proposed the hyperfiltration hypothesis, which states that insufficient nephron dosing might cause compensatory hyperfiltration and eventually result in glomerulosclerosis and allograft progressive damage (11). Several studies have been conducted to confirm this hypothesis, nevertheless, the results are contradictory (12-19).

Presently, nephron dosing cannot be measured directly in vivo, yet the allograft kidney weight and volume correlate with nephron dosing (20-22). Body weight, body surface area (BSA), and body mass index (BMI) can represent the metabolic demands of the recipients (23-25). Based on the above information, we adopted the ratio of donor kidney weight (DKW), donor kidney volume (DKV), donor body weight (DBW), donor BSA (DBSA) and recipient body weight (RBW), recipient BSA (RBSA), and recipient BMI (RBMI) as nephron dosing to the recipient's metabolic demands mismatch. Subsequently, we conducted a retrospective cohort study to determine the effect of donor nephron dosing on the recipient's metabolic demands mismatch on the outcomes of renal transplant recipients. We further explored its predictive value on the posttransplant renal function of recipients.

We present the following article in accordance with the STROBE reporting checklist (available at http://dx.doi. org/10.21037/tau-20-680).

\section{Methods}

\section{Study population}

This is a retrospective observational cohort and 290 recipients who received living relative renal transplantation in our center between December 2013 and December 2015 were included in our study. In order to limit other factors that might affect renal allograft function, our study population was restricted to living relative donor renal transplantation. Patients who received other solid organ simultaneous transplantation and dual en-bloc kidney transplantation were excluded. Patients with other conditions such as trauma and urinary tract obstruction leading to allograft impairment or loss were also excluded from our study. Our study was approved by the West China Hospital Institutional Review Board, Sichuan University (ChiCTR-POC-16008632: the registration number of ethics board) and informed consent was taken from all the patients. Our study was conducted in accordance with the Declaration of Helsinki (as revised in 2013).

\section{Data collection}

The donor and recipient data were collected at the inclusion time. Donor data included age, gender, height $(\mathrm{cm})$, weight $(\mathrm{kg})$, past history and renal function during the follow-up. Recipient data included age, gender, height $(\mathrm{cm})$, weight $(\mathrm{kg})$, history of organ transplantation, duration of dialysis, HLA mismatch, and the panel reactive antibody (PRA). The renal function trajectory and proteinuria were recorded during the follow-up at 1, 3, 6, 12, 18, 24, 30, and 36 months. Clinical related data included warm and cold ischemia time, renal allograft weight, volume after perfusion, and intraoperative complications. All patients took part in periodical outpatient follow-up.

The primary endpoints included a serum creatinine level and an estimated glomerular filtration rate (eGFR) at 1, 3, $6,12,18,24,30$, and 36 months after transplantation, and the survival of patient and graft at 1, 2, and 3 years. The secondary endpoints included the incidence of delayed graft function (DGF), rejection, infection, and proteinuria.

We use the modification of diet in renal disease equation to calculate eGFR: eGFR $\left(\mathrm{mL} / \mathrm{min} / 1.73 \mathrm{~m}^{2}\right)=186 \times($ serum creatinine level $)^{-1.154} \times(\text { age })^{-0.203} \times(0.742$ if female $) \times(1.212$ if African-American), where the serum creatinine level is expressed in $\mathrm{mg} / \mathrm{dL}$. The BSA was calculated using the Mostellar formula (26): BSA $\left(\mathrm{m}^{2}\right)=[$ weight $(\mathrm{kg}) \times$ height $(\mathrm{cm}) / 3,600]^{0.5}$. BMI $\left(\mathrm{kg} / \mathrm{m}^{2}\right)=$ weight $(\mathrm{kg}) /$ height $(\mathrm{m})^{2}$. DGF was defined as the need for dialysis within the first week after the transplantation. The diagnosis of rejection was based on the indication biopsy or clinical symptoms.

\section{Measurements of donor kidney morphology parameters}

All donors received living-relative retroperitoneal pure laparoscopic donor nephrectomies, which was conducted by the same transplant surgeon (T Lin) in our center. The donor kidney was immediately perfused after retiring. After reperfusion, the donor kidney was immediately weighed on the electronic weighting scale in the operating room. The renal volume was subsequently measured with a measuring glass. 


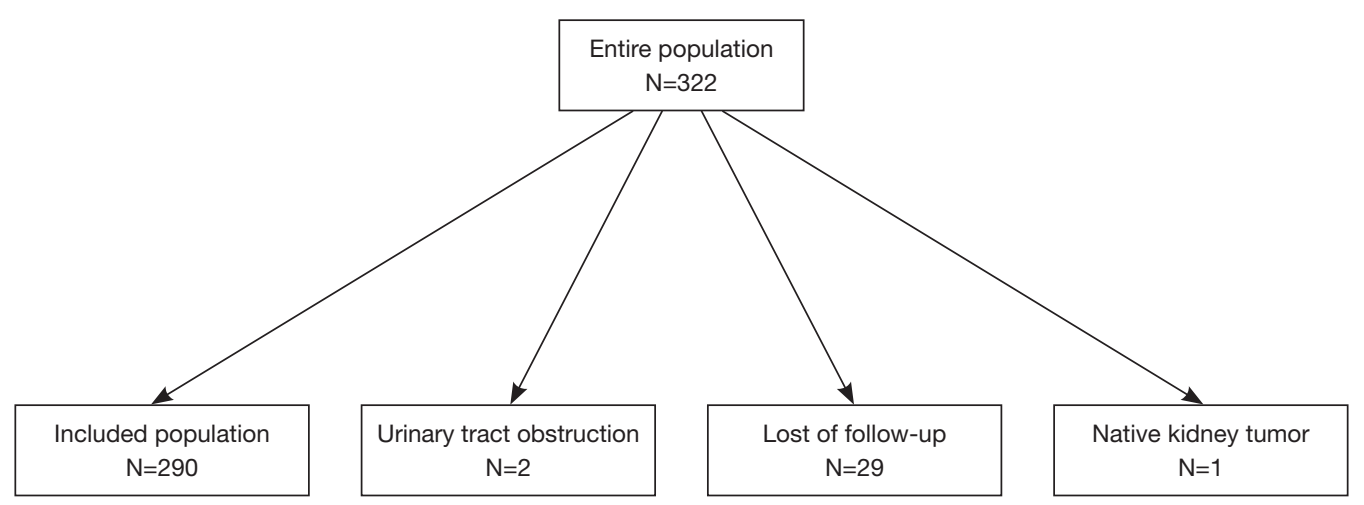

Figure 1 The flow chart of 322 living relative donor renal transplantation.

\section{Immunosuppression regimen}

Based on the immunological risk stratification, renal transplant recipients received different induction treatment strategies. All patients received standard triple immunosuppression, which consisted of steroids, tacrolimus (282 cases) or cyclosporine (8 cases), and mycophenolic acid. Daily dosages of tacrolimus or cyclosporine were administered orally, starting on the second day after transplantation, at a dose of 3 or $150 \mathrm{mg} /$ day, respectively. Mycophenolic acid was administered at 2,000 mg/day, starting the day before transplantation. The dose of the drug was individually adjusted, with the target trough blood level being between 5 and $10 \mathrm{ng} / \mathrm{mL}$, and $30 \mu \mathrm{g} \cdot \mathrm{h} / \mathrm{mL}$ and $60 \mu \mathrm{g} \cdot \mathrm{h} / \mathrm{mL}$ for tacrolimus and mycophenolic acid, respectively. Methylprednisolone was administered intravenously for three days after transplantation, and prednisone was taken orally from the fourth day at an initial dose of $60 \mathrm{mg} /$ day. The dose was reduced over time and was maintained at 5 or $10 \mathrm{mg} /$ day.

\section{Statistical analysis}

Categorical variables were analyzed with $\chi^{2}$-tests and continuous variables were compared using Student's $t$-tests or ANOVA. A nonparametric test was used for the median comparison. Univariate and multivariate linear regression analysis was used to evaluate the effect of nephron dosing on the recipient's metabolic demands mismatch on the renal allograft function. Survival analysis was performed using the Kaplan-Meier survival curves. The adjusted factors in the statistical analysis included age, gender, HLA mismatch, percentage of PRA category I and II, warm ischemia time, history of transplantation, duration of dialysis, induction treatment, the incidence of DGF, rejection, and infection. The threshold value for eGFR being $60 \mathrm{~mL} / \mathrm{min} / 1.73 \mathrm{~m}^{2}$ or above at 1 year after transplantation was determined using the receiver operating characteristic (ROC) curve. The results were considered statistically significant if the $\mathrm{P}$ value was less than 0.05 (two-tailed tests). All statistical procedures were performed using SPSS version 22.0 (IBM, USA) and SAS version 9.2 (North Carolina University, USA).

\section{Results}

\section{General characteristics of donors and recipients}

In this study, 290 participants were included from 322 living related renal transplantation donors and recipients (Figure 1). The general characteristics are shown in Tables 1 and 2, respectively. The preoperative renal function of all donors was considered normal. The serum creatinine and cystatin $\mathrm{C}$ at one and three months after operation increased significantly $(\mathrm{P}<0.01)$, but were still within the normal range. Most of the recipients were middle-aged males and $15(5.17 \%)$ patients received preemptive transplantations. Parameters of the surgery and donor kidney are shown in Table 2.

\section{Renal allograft function}

The eGFR of recipients at $1,3,6,12,18$, and 24 months after transplantation is $71.7 \pm 19.2,71.7 \pm 19.3,71.9 \pm 18.0$, $75.1 \pm 20.2,78.2 \pm 19.7$ and $76.5 \pm 22.3 \mathrm{~mL} / \mathrm{min} / 1.73 \mathrm{~m}^{2}$, respectively. The mean serum creatinine was within the normal range. The renal function of recipients in the first year after transplantation tended to be stable. According to the univariate linear regression analysis, eGFR at 1, 3, 6, 
Table 1 General characteristics of living kidney donors

\begin{tabular}{|c|c|}
\hline Donor-related factors & Number $(\%) /$ mean $\pm \mathrm{SD}$ \\
\hline Gender (male/female) & 95/195 (32.8\%/67.2\%) \\
\hline Age (year) & $48.3 \pm 8.7$ \\
\hline Side of donor kidney (left/right) & 258/32 (89.0\%/11.0\%) \\
\hline Height (cm) & $159.6 \pm 7.3$ \\
\hline Weight (kg) & $62.5 \pm 9.8$ \\
\hline Donor BSA $\left(\mathrm{m}^{2}\right)$ & $1.6 \pm 0.3$ \\
\hline $\begin{array}{l}\text { History of abdominal operation } \\
\text { (yes/no) }\end{array}$ & $47 / 243(16.2 \% / 83.8 \%)$ \\
\hline \multicolumn{2}{|l|}{ Serum creatinine $(\mu \mathrm{mol} / \mathrm{L})$} \\
\hline Pre-operation & $62.5 \pm 12.0$ \\
\hline 1-month post-operation & $99.1 \pm 20.2$ \\
\hline 3-month post-operation & $94.1 \pm 20.2$ \\
\hline \multicolumn{2}{|l|}{ Blood urea nitrogen (mmol/L) } \\
\hline Pre-operation & $5.5 \pm 1.6$ \\
\hline 1-month post-operation & $6.4 \pm 1.7$ \\
\hline 3-month post-operation & $6.0 \pm 1.4$ \\
\hline \multicolumn{2}{|l|}{ Cystatin C (mg/L) } \\
\hline Pre-operation & $0.9 \pm 0.1$ \\
\hline 1-month post-operation & $1.2 \pm 1.2$ \\
\hline 3-month post-operation & $1.1 \pm 0.2$ \\
\hline Length of stay & $8.4 \pm 1.7$ \\
\hline
\end{tabular}

BSA, body surface area.

12, and 24 months after transplantation was significantly correlated with DKW/RBW, DKW/RBSA, DKW/RBMI, DKV/RBW, DKV/RBSA, DKV/RBMI, DBW/RBW (Table 3). The serum creatinine at 6,12 , and 24 months post-transplantation significantly negatively correlated with DKW/RBW, DKW/RBSA, DKW/RBMI, DKV/ RBW, DKV/RBSA, DKV/RBMI, DBW/RBW (Table 4). The mean tacrolimus trough levels for $1,3,6,12,18$, and 24 months was $7.5 \pm 2.8,6.4 \pm 1.6,6.1 \pm 1.9,5.8 \pm 2.2,5.4 \pm 1.5$ and $5.3 \pm 1.4 \mathrm{ng} / \mathrm{mL}$, respectively. Adjusted and unadjusted multivariate linear regression analysis showed that, DKW/ $\mathrm{RBW}$, duration of dialysis, and the age of the donor were significantly correlated with eGFR 6 months after transplantation $(\mathrm{P}<0.01)$. DKW/RBW and the age of the donor were independent contributing factors for eGFR at 12,18 , and 24 months after transplantation. DKW/
Table 2 General characteristics of donor kidney and recipients included in the study

\begin{tabular}{|c|c|}
\hline Recipient-related factors & Number $(\%) /$ mean $\pm \mathrm{SD}$ \\
\hline Gender (male/female) & $222 / 68(76.6 \% / 23.4 \%)$ \\
\hline Age (year) & $33.0 \pm 9.0$ \\
\hline Height (cm) & $166.6 \pm 7.3$ \\
\hline Weight (kg) & $58.3 \pm 10.8$ \\
\hline Recipient BSA $\left(\mathrm{m}^{2}\right)$ & $1.6 \pm 0.2$ \\
\hline Recipient BMI $\left(\mathrm{kg} / \mathrm{m}^{2}\right)$ & $21.0 \pm 3.4$ \\
\hline History of transplant (yes/no) & $3 / 287(1.0 \% / 99.0 \%)$ \\
\hline Preemptive transplant, $\mathrm{n}(\%)$ & $15(5.2 \%)$ \\
\hline Duration of dialysis (month) & $13.4 \pm 12.7$ \\
\hline HLA mismatches $(0-8)$ & $3.7 \pm 1.4$ \\
\hline PRA I\% median (range) & $0(0-67)$ \\
\hline PRA II\% median (range) & $0(0-61)$ \\
\hline Preoperative serum creatinine $(\mu \mathrm{mol} / \mathrm{L})$ & $1,045.0 \pm 342.6$ \\
\hline Preoperative cystatin C (mg/L) & $7.5 \pm 2.0$ \\
\hline \multicolumn{2}{|l|}{ Induction therapy } \\
\hline Anti-CD25 monoclonal antibody & $221(76.2 \%)$ \\
\hline Antithymocyte globulin & $24(8.3 \%)$ \\
\hline None & $45(15.5 \%)$ \\
\hline Warm ischemia time (sec) & $185.9 \pm 61.2$ \\
\hline Cold ischemia time (min) & $129.3 \pm 35.4$ \\
\hline Kidney weight after perfusion (g) & $180.1 \pm 33.9$ \\
\hline Kidney volume after perfusion (mL) & $157.6 \pm 34.9$ \\
\hline DKW/RBW (g/kg) & $3.2 \pm 0.7$ \\
\hline DKW/RBSA $\left(\mathrm{g} / \mathrm{m}^{2}\right)$ & $110.6 \pm 20.9$ \\
\hline DKW/RBMI $\left(\mathrm{g} / \mathrm{kg} / \mathrm{m}^{2}\right)$ & $8.8 \pm 2.0$ \\
\hline DKV/RBW (mL/kg) & $2.8 \pm 0.7$ \\
\hline DKV/RBSA $\left(\mathrm{mL} / \mathrm{m}^{2}\right)$ & $96.7 \pm 21.1$ \\
\hline DKV/RBMI $\left(\mathrm{mL} / \mathrm{kg} / \mathrm{m}^{2}\right)$ & $7.7 \pm 1.9$ \\
\hline DBW/RBW & $1.1 \pm 0.2$ \\
\hline DBSA/RBSA & $1.0 \pm 0.2$ \\
\hline
\end{tabular}

BSA, body surface area; BMI, body mass index; HLA, human leukocyte antigen; PRA, panel reactive antibody; DKW, donor kidney weight; RBW, recipient body weight; RBSA, recipient body surface area; RBMI, recipient body mass index; DKV, donor kidney volume; DBW, donor body weight; DBSA, donor body surface area. 
Table 3 Univariate linear regression analysis of eGFR within 2 year after transplantation

\begin{tabular}{|c|c|c|c|c|c|c|c|c|c|c|}
\hline & \multicolumn{2}{|c|}{1 month } & \multicolumn{2}{|c|}{3 months } & \multicolumn{2}{|c|}{6 months } & \multicolumn{2}{|c|}{12 months } & \multicolumn{2}{|c|}{24 months } \\
\hline DKW/RBSA & 0.4 & $<0.01$ & 0.4 & $<0.01$ & 0.4 & $<0.01$ & 0.4 & $<0.01$ & 0.5 & $<0.01$ \\
\hline DKV/RBSA & 0.3 & $<0.01$ & 0.3 & $<0.01$ & 0.4 & $<0.01$ & 0.3 & $<0.01$ & 0.4 & $<0.01$ \\
\hline DKV/RBMI & 0.3 & $<0.01$ & 0.3 & $<0.01$ & 0.4 & $<0.01$ & 0.3 & $<0.01$ & 0.4 & $<0.01$ \\
\hline DBW/RBW & 0.4 & $<0.01$ & 0.4 & $<0.01$ & 0.4 & $<0.01$ & 0.5 & $<0.01$ & 0.4 & $<0.01$ \\
\hline DKeGFR/(DKVRBMI) & 0.2 & $<0.01$ & 0.2 & $<0.01$ & 0.2 & 0.01 & 0.1 & 0.2 & 0.03 & 0.8 \\
\hline DKeGFR/(DKWRBW) & 0.3 & $<0.01$ & 0.3 & $<0.01$ & 0.3 & $<0.01$ & 0.2 & $<0.01$ & 0.1 & 0.3 \\
\hline DKeGFR/(DKW*RBSA) & 0.3 & $<0.01$ & 0.2 & $<0.01$ & 0.3 & $<0.01$ & 0.2 & 0.02 & 0.1 & 0.6 \\
\hline DKeGFR/(DKW*RBMI) & 0.2 & $<0.01$ & 0.2 & $<0.01$ & 0.2 & $<0.01$ & 0.2 & 0.02 & 0.01 & 0.5 \\
\hline
\end{tabular}

eGFR, estimated glomerular filtration rate; DKW, donor kidney weight; RBW, recipient body weight; RBSA, recipient body surface area; RBMI, recipient body mass index; DKV, donor kidney volume; DBW, donor body weight; DBSA, donor body surface area; DKeGFR, donor kidney eGFR.

RBW, donor and recipient's age, sex, and PRA significantly correlated with the serum creatinine at 6 and 12 months after transplantation $(\mathrm{P}<0.05)$.

We identified several new indexes representing nephron dosing, such as donor kidney volume density (eGFR/volume of the donor kidney) and donor kidney mass density (eGFR/ mass of the donor kidney). These indexes to the metabolic demand mismatch significantly correlated with eGFR and serum creatinine within six months after transplantation; nevertheless, it did not show a greater correlation (Tables 3 and 4).

\section{Effectiveness of Indexes}

The effectiveness of indexes to predict a good renal allograft function (eGFR $\geq 60 \mathrm{~mL} / \mathrm{min} / 1.73 \mathrm{~m}^{2}$ ) 12 months after transplantation is shown in Table 5. Among these indexes, DKW/RBW had the largest areas under the ROC curve of 0.655 and the best prediction performance. According to the multivariate analysis, the age of the donor also affected the postoperative renal function. This was the first time we compared the AUC of different indexes after stratification according to the age of the donor (Table 5). When the age of the donor was 55 years or more, the area under the ROC curve of every index increased and the prediction performance enhanced. The AUC of DKW/RBW reached a maximum of 0.76 .

\section{Subgroup analysis}

According to the donor age, all renal transplant recipients were divided into two groups: the young group and ( $<55$ years) and the old group (55 years and above). Each group was also divided into tertiles according to the DKW/ RBW: low (<2.83), medium (2.83-3.35), and high $(\geq 3.35)$ in the young group; low (<2.81), medium (2.81-3.41), and high ( $\geq 3.41)$ in the old group. In the young and old groups, the baseline characteristics, including the age of the donor, preoperative serum creatinine level, warm ischemia time, dialysis time, HLA mismatch, PRA, and induction therapy etc., were comparable among the three groups. In the young and old groups, the tacrolimus trough level during 
Table 4 Univariate linear regression analysis of the serum creatinine within 2 year after transplantation

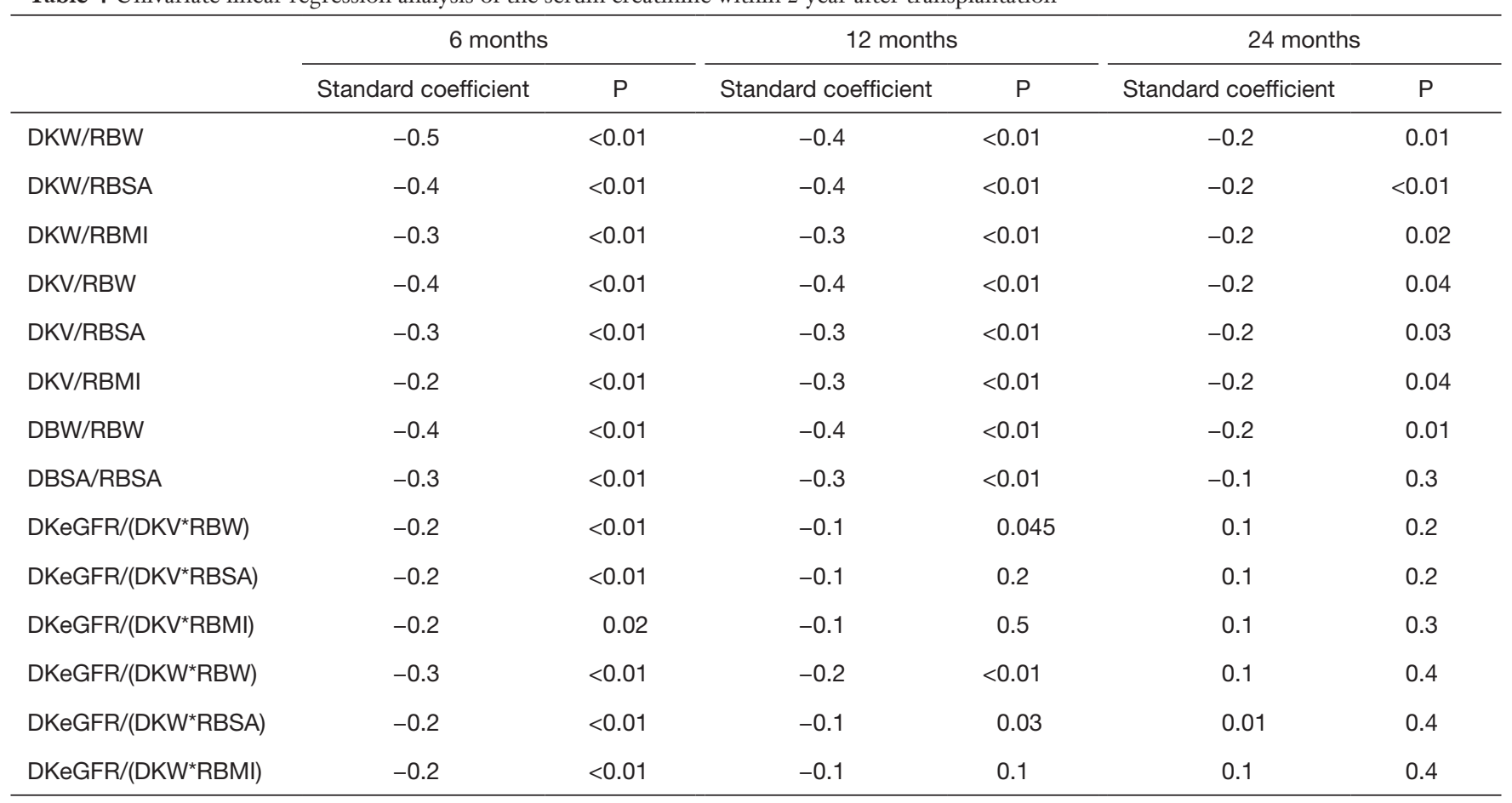

DKW, donor kidney weight; RBW, recipient body weight; RBSA, recipient body surface area; RBMI, recipient body mass index; DKV, donor kidney volume; DBW, donor body weight; DBSA, donor body surface area; DKeGFR, donor kidney eGFR.

the follow-up period showed no significant difference among the three groups.

In the young and old groups, there was no significant difference between the DGF and infection during the follow-up period among the three groups. In our study, the overall rejection rate was $17.93 \%(52 / 290)$ and the overall biopsy-proven acute rejection rate was $3.45 \%$ (10/290). The number of recipients who were rejected in the high group was significantly lower than that in the low and medium groups (young group: high vs. medium vs. low, $11.8 \%$ vs. $16.2 \%$ vs. $27.0 \%, \mathrm{P}<0.05$; old group: high vs. medium vs. low, $0 \%$ vs. $20 \%$ vs. $36.8 \%, \mathrm{P}=0.008$ ). Whether the donor was young or old, the renal function of the recipients improved as the DKW/RBW increased. The eGFR of recipients in the high group within 2 years after transplantation was significantly higher than that of the other groups. The urine protein of the recipients is shown in Table 6, with no significant difference 2 years after transplantation among the three groups.

\section{Survival analysis}

In our study, the 1, 2, and 3-year graft survival rates were
$100 \%, 98.8 \%$, and $96.2 \%$ respectively. Four recipients had renal allograft failure. The 1, 2, and 3-year patient survival rates were $98 \%, 97 \%$, and $97 \%$, respectively. Seven patients died due to infection. The 1, 2, and 3-year graft and patient survival rates were comparable among these groups $(\mathrm{P}>0.05)$.

\section{Prediction of renal function}

According to the age of the donor, we calculated the respective threshold value of $\mathrm{DKW} / \mathrm{RBW}$, which can predict the eGFR $\geq 60 \mathrm{~mL} / \mathrm{min} / 1.73 \mathrm{~m}^{2} 12$ months after transplantation. When the age of the donor was less than 55 years, the threshold value of DKW/RBW was $2.61 \mathrm{~g} / \mathrm{kg}$. The threshold value was $3.09 \mathrm{~g} / \mathrm{kg}$ when the age of the donor was 55 years and above. When the DKW/RBW ratio exceeded the threshold value, the recipients most likely had a good renal function (eGFR $\geq 60 \mathrm{~mL} / \mathrm{min} / 1.73 \mathrm{~m}^{2}$ ) 12 months after transplantation.

\section{Discussion}

Owing to the increasing shortage of donor kidneys, 
Table 5 The areas under the ROC curve of indexes to predict the eGFR $\geq 60 \mathrm{~mL} / \mathrm{min} / 1.73 \mathrm{~m} 2$ at 12 months after transplantation according to the stratified analyses by donor age

\begin{tabular}{|c|c|c|c|}
\hline & \multirow[b]{2}{*}{ ROC-AUC } & \multicolumn{2}{|c|}{ ROC-AUC } \\
\hline & & $\begin{array}{c}\text { Donor } \\
<55 \text { years old }\end{array}$ & $\begin{array}{l}\geq 55 \text { years } \\
\quad \text { old }\end{array}$ \\
\hline DKW/RBW & 0.66 & 0.62 & 0.76 \\
\hline DKW/RBSA & 0.64 & 0.62 & 0.71 \\
\hline DKW/RBMI & 0.62 & 0.59 & 0.70 \\
\hline DKV/RBW & 0.63 & 0.58 & 0.74 \\
\hline DKV/RBSA & 0.61 & 0.57 & 0.71 \\
\hline DKV/RBMI & 0.60 & 0.54 & 0.72 \\
\hline DBW/RBW & 0.65 & 0.62 & 0.74 \\
\hline DBSA/RBSA & 0.65 & 0.63 & 0.74 \\
\hline DKeGFR/(DKVRBW) & 0.59 & 0.53 & 0.65 \\
\hline DKeGFR/(DKVRBSA) & 0.58 & 0.54 & 0.61 \\
\hline DKeGFR/(DKV*RBMI) & 0.59 & 0.53 & 0.63 \\
\hline DKeGFR/(DKW*RBW) & 0.61 & 0.53 & 0.68 \\
\hline DKeGFR/(DKW*RBSA) & 0.59 & 0.52 & 0.65 \\
\hline DKeGFR/(DKW*RBMI) & 0.59 & 0.52 & 0.64 \\
\hline
\end{tabular}

eGFR, estimated glomerular filtration rate; DKW, donor kidney weight; RBW, recipient body weight; RBSA, recipient body surface area; RBMI, recipient body mass index; DKV, donor kidney volume; DBW, donor body weight; DBSA, donor body surface area; DKeGFR, donor kidney eGFR; AUC, areas under the curve.

transplant physicians must expand the organ donor pool and improve the long-term survival of renal transplant recipients. Nephron dosing to the recipient's metabolic demands mismatch, as the prognostic factor affecting the allograft renal function, has drawn the attention and interest of transplant physicians $(11,27)$. This single-center cohort study included 290 living relative renal transplantation donors and recipients with a median follow-up of 22 months (range: 1-36 months). We found that nephron dosing to the recipient's metabolic demands mismatch, especially DKW/ RBW and the age of the donor, were significantly associated with the renal function within2 years after transplant in renal transplant recipients.

In this study, $67.24 \%$ of donors were female, and $76.55 \%$ of recipients were male. Generally, males have a greater $\mathrm{BMI}$ and BSA and are associated with a greater kidney weight and volume $(14,28,29)$. According to our study, the ratio of the nephron and recipient's metabolism demands had an extremely significant linear correlation with the early renal function in transplant recipients. Relative to recipient metabolic demands, recipients that had more nephron had a better early allograft renal function. Multivariate analysis found that the age of the donor and DKW/RBW were the predictors of the early postoperative renal function in recipients. Moreover, an early postoperative renal function could predict the long-term renal function after renal transplantation (30). In addition, we identified new indexes that represented nephron dosing, such as the donor kidney volume density (eGFR/volume of the donor kidney) and donor kidney mass density (eGFR/mass of the donor kidney). These indexes to metabolic demands mismatch correlated significantly linearly with the early renal function in the recipients. In our study, recipients with a high DKW/ RBW experienced less rejection. Nonetheless, further research needs to be conducted to exclude confounding factors.

This is the first time we combined donor age and nephron dosing to the recipient's metabolic demands mismatch. Stratification analysis on the age of the donor found that the effectiveness of the indexes to predict a good renal allograft function enhanced when the age of the donor age exceeded 55 years. Therefore, we endeavor to focus more on nephron dosing to the recipient's metabolic demands mismatch in elderly donors. According to a previous study, hyperfiltration could cause glomerular damage and sclerosis and lead to proteinuria (11). However, recipients in our cohort showed no symptoms of glomerular sclerosis. This may be due to our shorter follow-up duration. In our cohort, four recipients had renal allograft failure and seven recipients died. However, nephron dosing to the recipient's metabolic demands mismatch was not the risk factor behind the short-term patient and graft survival. The main causes of graft loss and death were still rejection and infection. Thus, a long-term follow-up duration is needed to determine the impact on long-term survival. We determined the thresholds of DKW/RBW under different donor ages. Comprehensive analysis of the age of the donor and relative nephron was more advantageous to predict the renal function in kidney transplant recipients. When the age of the donor was less than 55 years, the DKW/RBW threshold was significantly lower. However, when the age of the donor was 55 years and above, the threshold of DKW/ $\mathrm{RBW}$ was $3.09 \mathrm{~g} / \mathrm{kg}$.

Though many previous studies had investigated nephron dosing to the recipient's metabolic demands mismatch, 
Table 6 Compare of urine protein in recipients according to the stratified analyses by donor age [median (ranges)]

\begin{tabular}{lcccrr}
\hline & Donor age & Low & Medium & High \\
\hline 1 year & $<55$ & $0.2(0-3.0)$ & $0.1(0-6.0)$ & $0.1(0-1.0)$ & 0.3 \\
& $\geq 55$ & $0.1(0-1.0)$ & $0.1(0-0.7)$ & $0.1(0-0.5)$ & 0.6 \\
2 year & $<55$ & $0.1(0-2.0)$ & $0.1(0-6.0)$ & $0.1(0-2.0)$ & $0.1(0-0.3)$ \\
& $\geq 55$ & $0.1(0-0.7)$ & $0.2(0-0.7)$ & 0.6 \\
\hline
\end{tabular}

there was no consensus among various research results. Brenner et al. proposed the hyperfiltration theory in 1992 and stipulated that the ratio of donor kidney weight and the recipient body weight could impact the long-term survival in recipients (11). Since then, transplant physicians globally have begun to focus more on relative nephron, yet their research conclusions have been inconsistent. Kim et al. found that DKW/RBW was linearly correlated with the 3 -year creatinine, creatinine clearance, and urine protein in kidney transplant recipients (12). This study included 82 living donor kidney transplant recipients and the sample size was too small. Giral et al. reported that recipients with low DKW/RBW experienced the decline of GFR (glomerular filtration rate) 7 years after transplantation, and had a higher risk of proteinuria and were taking more antihypertensive agents. The risk of a 2-year graft loss increased by $55 \%$ (13). This prospective, multicenter cohort study consisted of 1,053 deceased donor kidney transplant recipients who had a longer ischemia time and higher risk of organ injury during procurement. However, Tent et al. investigated 293 living renal transplantation and had shown that recipients with low donor/recipient BSA experienced an increase in GFR after transplantation. Nonetheless, no differences were recorded on the renal function 5 years after transplantation (14), which did not support the hypothesis. Dinis et al. reported that DKW/RBW, DKV/RBW, and DBW/RBW did not correlate with the renal function within 5 years after transplantation in 236 deceased kidney transplant recipients. Furthermore, there was no significant difference in DGF, rejection, and the 5-year patient and graft survival (17).

Our study had a larger sample size of 290 living kidney transplantation. All laparoscopic donor nephrectomies were completed by the same doctor (Dr. Lin); this eliminated surgery-related confounding factors. This single-center study eliminated center-related bias as much as possible. However, our study had several limitations. First, our follow-up duration was short, and could not determine the long-term impact of the relative nephron. Second, we did not compare the direct measurement method and preoperative imaging method by acquiring data about renal mass and volume. Third, most of the recipients did not receive biopsy, and the allograft pathological changes were not determined.

\section{Conclusions}

In conclusion, nephron dosing to the recipient's metabolic demands mismatch combined with the age of the donor has a significant impact on the early post-transplant renal function in renal transplant recipients. However, further studies with larger sample sizes and longer follow-up times are urgently needed.

\section{Acknowledgments}

The authors acknowledge the collaboration of the transplantation team at West China Hospital, Sichuan University.

Funding: This study was collectively supported by grants from the National Natural Science Foundation of China (81870513,81470980, 81600584), Sichuan Science and Technology Program (2019YJ0133), the Fundamental Research Funds for the Central Universities (2017SCU11022), and 1.3.5 Project for Disciplines of Excellence-Clinical Research Incubation Project, West China Hospital, Sichuan University (2018HXFH049, ZY2016104). The funders had no role in study design, data collection or analysis, preparation of the manuscript, or the decision to publish.

\section{Footnote}

Reporting Checklist: The authors have completed the STROBE reporting checklist. Available at http://dx.doi. 
org/10.21037/tau-20-680

Data Sharing Statement: Available at http://dx.doi. org/10.21037/tau-20-680

Peer Review File: Available at http://dx.doi.org/10.21037/ tau-20-680

Conflicts of Interest: All authors have completed the ICMJE uniform disclosure form (available at http://dx.doi. org/10.21037/tau-20-680). The authors have no conflicts of interest to declare.

Ethical Statement: The authors are accountable for all aspects of the work in ensuring that questions related to the accuracy or integrity of any part of the work are appropriately investigated and resolved. Our study was approved by the West China Hospital Institutional Review Board, Sichuan University (registration number-ChiCTRPOC-16008632) and informed consent was taken from all the patients. Our study was conducted in accordance with the Declaration of Helsinki (as revised in 2013).

Open Access Statement: This is an Open Access article distributed in accordance with the Creative Commons Attribution-NonCommercial-NoDerivs 4.0 International License (CC BY-NC-ND 4.0), which permits the noncommercial replication and distribution of the article with the strict proviso that no changes or edits are made and the original work is properly cited (including links to both the formal publication through the relevant DOI and the license). See: https://creativecommons.org/licenses/by-nc-nd/4.0/.

\section{References}

1. Hariharan S, Johnson CP, Bresnahan BA, et al. Improved graft survival after renal transplantation in the United States, 1988 to 1996. N Engl J Med 2000;342:605-12.

2. Abecassis M, Bartlett ST, Collins AJ, et al. Kidney transplantation as primary therapy for end-stage renal disease: a National Kidney Foundation/Kidney Disease Outcomes Quality Initiative (NKF/KDOQITM) conference. Clin J Am Soc Nephrol 2008;3:471-80.

3. Terasaki PI, Cecka JM, Gjertson DW, et al. High survival rates of kidney transplants from spousal and living unrelated donors. N Engl J Med 1995;333:333-6.

4. Axelrod DA, McCullough KP, Brewer ED, et al. Kidney and pancreas transplantation in the United States,
1999-2008: the changing face of living donation. Am J Transplant 2010;10:987-1002.

5. Baid-Agrawal S, Frei UA. Living donor renal transplantation: recent developments and perspectives. Nat Clin Pract Nephrol 2007;3:31-41.

6. Meier-Kriesche HU, Schold JD, Kaplan B. Long-term renal allograft survival: have we made significant progress or is it time to rethink our analytic and therapeutic strategies? Am J Transplant 2004;4:1289-95.

7. Morrison AB. Experimentally induced chronic renal insufficiency in the rat. Lab Invest 1962;11:321-32.

8. Shimamura T, Morrison AB. A progressive glomerulosclerosis occurring in partial five-sixths nephrectomized rats. Am J Pathol 1975;79:95-106.

9. Hostetter TH, Olson JL, Rennke HG, et al. Hyperfiltration in remnant nephrons: a potentially adverse response to renal ablation. J Am Soc Nephrol 2001;12:1315-25.

10. Azuma H, Nadeau K, Mackenzie HS, et al. Nephron mass modulates the hemodynamic, cellular, and molecular response of the rat renal allograft. Transplantation 1997;63:519-28.

11. Brenner BM, Cohen RA, Milford EL. In renal transplantation, one size may not fit all. J Am Soc Nephrol 1992;3:162-9.

12. Kim YS, Moon JI, Kim DK, et al. Ratio of donor kidney weight to recipient bodyweight as an index of graft function. Lancet 2001;357:1180-1.

13. Giral M, Foucher Y, Karam G, et al. Kidney and recipient weight incompatibility reduces long-term graft survival. J Am Soc Nephrol 2010;21:1022-9.

14. Tent H, Lely AT, Toering TJ, et al. Donor kidney adapts to body dimensions of recipient: no influence of donor gender on renal function after transplantation. Am J Transplant 2011;11:2173-80.

15. Sikora MB, Shaaban A, Beddhu S, et al. Effect of donor kidney volume on recipient outcome: does the "dose" matter? Transplantation 2012;94:1124-30.

16. Dar TI, Tyagi V, Pahwa M, et al. A study to evaluate the effect of ratio of donor kidney weight to recipient body weight on renal graft function. Urol Ann 2014;6:139-41.

17. Dinis P, Nunes P, Marconi L, et al. Small kidneys for large recipients: does size matter in renal transplantation? Transplant Proc 2015;47:920-5.

18. Tanriover B, Fernandez S, Campenot ES, et al. Live Donor Renal Anatomic Asymmetry and Posttransplant Renal Function. Transplantation 2015;99:e66-74.

19. Garcia-Covarrubias L, Pliego C, Bermudez L, et al. 
Correlation of Allograft Weight to Recipient Body Weight Index on Renal Function in Kidney Transplantation.

Transplant Proc 2016;48:578-82.

20. Luyckx VA, Brenner BM. The clinical importance of nephron mass. J Am Soc Nephrol 2010;21:898-910.

21. Nyengaard JR, Bendtsen TF. Glomerular number and size in relation to age, kidney weight, and body surface in normal man. Anat Rec 1992;232:194-201.

22. Nishimura Y, Tomikawa S, Beck Y, et al. Kidney graft weight as an important risk factor for long-term graft survival. Transplant Proc 1998;30:107-10.

23. Meier-Kriesche HU, Vaghela M, Thambuganipalle R, et al. The effect of body mass index on long-term renal allograft survival. Transplantation 1999;68:1294-7.

24. Pirsch JD, Armbrust MJ, Knechtle SJ, et al. Obesity as a risk factor following renal transplantation. Transplantation 1995;59:631-3.

25. Moreso F, Seron D, Anunciada AI, et al. Recipient body

Cite this article as: Qiu Y, Liu J, Jiang Y, Song T, Huang Z, Fan Y, Wang X, Lin T. Effect of donor kidney morphology parameters on the prognosis in living kidney transplantation recipients. Transl Androl Urol 2020;9(5):1957-1966. doi:10.21037/ tau-20-680 surface area as a predictor of posttransplant renal allograft evolution. Transplantation 1998;65:671-6.

26. Mosteller RD. Simplified calculation of body-surface area. N Engl J Med 1987;317:1098.

27. Brenner BM, Milford EL. Nephron underdosing: a programmed cause of chronic renal allograft failure. Am J Kidney Dis 1993;21:66-72.

28. Oh CK, Lee BM, Jeon KO, et al. Gender-related differences of renal mass supply and metabolic demand after living donor kidney transplantation. Clin Transplant 2006;20:163-70.

29. Poggio ED, Hila S, Stephany B, et al. Donor kidney volume and outcomes following live donor kidney transplantation. Am J Transplant 2006;6:616-24.

30. Hariharan S, McBride MA, Cherikh WS, et al. Posttransplant renal function in the first year predicts longterm kidney transplant survival. Kidney Int 2002;62:311-8. 